\section{ORIGINAL RESEARCH}

\author{
G.C. Chiang
}

W. Zhan

N. Schuff

M.W. Weiner

\title{
White Matter Alterations in Cognitively Normal apoE $\varepsilon 2$ Carriers: Insight into Alzheimer Resistance?
}

\begin{abstract}
BACKGROUND AND PURPOSE: The basis for decreased vulnerability to AD among apoE $\varepsilon 2$ carriers is unknown. The purpose of this study was to use diffusion tensor imaging to detect possible differences in white matter integrity between cognitively normal elderly apoE $\varepsilon 2$ carriers and apoE $\varepsilon 3 / \varepsilon 3$ controls.
\end{abstract}

MATERIALS AND METHODS: Thirty-nine cognitively normal elderly individuals (19 heterozygous carriers of the apoE $\varepsilon 2$ allele, 20 apoE $\varepsilon 3 / \varepsilon 3$ subjects as controls) underwent diffusion tensor MR imaging on a $4 \mathrm{~T}$ scanner. Fractional anisotropy, MD, and axial and radial diffusivity were compared using a ROI approach. In addition, an exploratory whole-brain analysis of fractional anisotropy between the 2 groups was undertaken using TBSS.

RESULTS: apoE $\varepsilon 2$ carriers had higher FA in the posterior cingulate white matter $(P=.01)$ and anterior corpus callosum $(P=.005)$ than apoE $\varepsilon 3 / \varepsilon 3$ controls, secondary to lower radial diffusivity. No significant differences in the FA of the posterior corpus callosum, anterior cingulate white matter, or parahippocampal white matter were seen. Whole-brain TBSS analysis detected regions of higher FA in the apoE $\varepsilon 2$ group in the superior longitudinal fasciculus, right thalamus, and the bilateral anterior limbs of the internal capsule, in addition to the posterior cingulum and corpus callosum $(P<.005)$. There were no regions in which the apoE $\varepsilon 3 / \varepsilon 3$ group had higher FA.

CONCLUSIONS: apoE $\varepsilon 2$ carriers harbor more robust white matter integrity that may be associated with decreased vulnerability to developing AD. This provides further evidence that regional DTI metrics may serve as early imaging biomarkers of $A D$ risk.

ABBREVIATIONS: $A D=$ Alzheimer disease; $a p o E=$ apolipoprotein $E ; F A=$ fractional anisotropy; $\mathrm{MD}=$ mean diffusivity; MMSE = Mini-Mental State Examination; TBSS = tract-based spatial statistics

T he 3 alleles of the apoE gene are associated with varying future risk of developing $\mathrm{AD}$, though the biologic basis for this relationship is unknown. ${ }^{1,2}$ Whereas apoE $\varepsilon 4$ carriers have an increased lifetime risk for $\mathrm{AD},{ }^{2}$ most of the population carries the apoE $\varepsilon 3 / \varepsilon 3$ genotype and $8 \%$ of the population carry an apoE $\varepsilon 2$ allele, ${ }^{3}$ which is believed to decrease the risk of developing AD. ${ }^{1}$

One potential approach to better understanding the biologic impact of apoE $\varepsilon 2$ is through neuroimaging. Specifically, DTI is an MR imaging technique that is sensitive to directional diffusivity of water in tissue, thereby noninvasively capturing microstructural white matter alterations in the brain. ${ }^{4}$ The directionality of water diffusion is most commonly expressed as FA, which is a scalar metric summarizing the shape of the diffusion tensor and calculated from the 3 orthogonal tensor eigenvalues in each image voxel $\left(\lambda_{1}, \lambda_{2}, \lambda_{3}\right)$. FA values can range, theoretically, from 0 (isotropic spherical diffusion) to 1 (complete anisotropic diffusion along a single direction), with high values suggesting well-oriented white matter tracts. MD

Received September 18, 2011; accepted after revision November 2.

From the Center for Imaging of Neurodegenerative Diseases, Department of Veteran Affairs Medical Center, San Francisco, California, and the Department of Radiology, University of California, San Francisco, California.

Please address correspondence to Gloria C. Chiang, MD, Department of Radiology, University of California at San Francisco, 505 Parnassus Ave M-391, San Francisco, CA 94143; e-mail: gloria.chiang@ucsf.edu

Indicates open access to non-subscribers at www.ajnr.org

http://dx.doi.org/10.3174/ajnr.A2984 is the average of the 3 eigenvalues. The diffusion tensor can be further decomposed into axial diffusivity $\left(\lambda_{1}\right)$, the diffusion coefficient in the direction of maximal diffusion, and radial diffusivity $\left(\left[\lambda_{2}+\lambda_{3}\right] / 2\right)$, the average of the 2 orthogonal diffusion coefficients. Animal studies have suggested that decreased FA dominated by axial diffusivity reflects wallerian degeneration and axonal loss, whereas decreased FA dominated by increased radial diffusivity reflects myelin breakdown, though this has yet to be proved in humans. ${ }^{5,6}$ We evaluated axial and radial diffusivity to potentially gain more specificity with respect to the underlying microstructural alterations.

Several studies applying DTI to AD subjects have found white matter degeneration manifesting primarily as decreased FA in the posterior cingulate white matter. ${ }^{7,8}$ Decreased FA has also been reported in the hippocampus, parahippocampal white matter, and frontoparietal white matter, though this is less consistently observed. ${ }^{9-12}$ A few studies have applied DTI to asymptomatic individuals with a high risk of developing future $\mathrm{AD}$ - namely, apoE $\varepsilon 4$ carriers-and found decreased FA in the cingulate white matter, parahippocampal white matter, posterior corpus callosum, and inferior longitudinal fasciculus. ${ }^{13-15}$ However, no known studies, to date, have described white matter changes specific to the apoE $\varepsilon 2$ allele.

The purpose of this study was to determine whether healthy apoE $\varepsilon 2$ carriers demonstrate microstructural white matter alterations, reflected by DTI metrics, compared with noncarriers. Specifically, we hypothesized that apoE $\varepsilon 2$ carriers would have higher FA values compared with apoE $\varepsilon 3 / \varepsilon 3$ 


\begin{tabular}{|c|c|c|c|}
\hline \multicolumn{4}{|l|}{ Baseline group characteristics } \\
\hline & apoE 2/3 & apoE 3/3 & $P$ Value \\
\hline $\bar{N}$ & 19 & 20 & \\
\hline Age (years) & $68(11.6)$ & $69(10.3)$ & 0.9 \\
\hline Gender (male:female) & $10: 9$ & $9: 11$ & 0.8 \\
\hline \multicolumn{4}{|l|}{ CVLT ॥ } \\
\hline Immediate recall total ST & $56(10)$ & $59(9)$ & 0.2 \\
\hline Short free recall ST & $0.6(1.3)$ & $0.8(1.1)$ & 0.8 \\
\hline Long free recall ST & $0.4(1.4)$ & $0.8(0.5)$ & 0.9 \\
\hline Delayed recall discriminability ST & $1.3(1.4)$ & $1.9(0.8)$ & 0.3 \\
\hline White matter lesion load (0:1:2) & 3:8:8 & $8: 7: 5$ & 0.3 \\
\hline
\end{tabular}

Data shown are means (standard deviations). White matter lesion load: $0=$ none, $1=$ mild, $2=$ moderate. CVLT indicates California Verbal Learning Test; ST indicates standard score.

controls in white matter tracts believed to be affected in $\mathrm{AD}$, particularly the posterior cingulate white matter. We further examined whether group differences in FA reflected differences in primarily axial or radial diffusivity. Finally, we performed an exploratory whole-brain voxel-based analysis to determine whether other regions of the brain showed significant FA differences between groups.

\section{Materials and Methods}

\section{Subjects}

Thirty-nine cognitively normal individuals, between the ages of 49 and 90, participated in this study: 19 were heterozygous for the apoE $\varepsilon 2$ allele, and the 20 controls were homozygous for the apoE $\varepsilon 3$ allele (Table). Written informed consent was obtained by all participants, and the study was approved by the institutional review boards of the University of California, San Francisco and the San Francisco Veteran Affairs Hospital. Study participants were included based on apoE genotype and matched by age. Thirty-five of the 39 participants were recruited from the San Francisco community via flyers, online bulletin boards, and word of mouth, then underwent neuropsychological assessment at the San Francisco Veteran Affairs Hospital to confirm normal cognitive functioning. Four were recruited by the University of California, San Francisco and San Francisco Veteran Affairs Hospital memory clinics as cognitively normal individuals. In addition, following informed consent, the physicians of all participants were contacted for known clinical diagnoses, and caregivers or family members were contacted to determine the participants' daily level of functioning. All participants lacked major neurologic or psychiatric illness and scored at least 27 of 30 on the MMSE. ${ }^{16}$

\section{apoE Genotyping}

All participants underwent apoE genotyping. Approximately $10 \mathrm{~mL}$ of blood was obtained from each participant in an ethylenediaminetetraacetic acid tube, gently mixed by inversion, and shipped at ambient temperature to an outside laboratory (Athena Diagnostics) for analysis within 24 hours of collection. The analysis was performed by restriction endonuclease digestion of a polymerase chain reactionamplified DNA fragment of the apoE gene.

\section{Data Acquisition}

Diffusion tensor imaging data were acquired on a 4T MR imaging scanner (MedSpec system; Bruker/Siemens, Erlangen, Germany), using a twice-refocused spin-echo echoplanar pulse sequence $(\mathrm{TR}=$ $6000 \mathrm{~ms}$, TE $=77 \mathrm{~ms}$ ). Images through the whole brain were acquired with 40 contiguous 3 -mm-thick sections, $128 \times 112$ matrix size, and a factor 2 generalized autocalibrating partially parallel acquisition ac- celeration to reduce geometric distortions, resulting in a spatial resolution of $2 \times 2 \times 3 \mathrm{~mm}$. A reference image $(b=0)$ and 6 diffusionweighted images $\left(b=800\right.$ seconds $/ \mathrm{mm}^{2}$, along 6 noncollinear directions) were acquired, using 4 averages to boost signal intensity-to-noise.

Given the possible confounding effects of vascular lesions, FLAIR images were acquired on the same $4 \mathrm{~T}$ MR imaging scanner to evaluate white matter hyperintensities. Imaging parameters were TR/TE/TI = $7000 \mathrm{~ms} / 400 \mathrm{~ms} / 2050 \mathrm{~ms}$ with rapid echo readouts, flip angle $=180^{\circ}$, and matrix size $=256 \times 192$, yielding $1 \times 1 \times 1 \mathrm{~mm}$ spatial resolution.

\section{Image Preprocessing}

FLAIR images for all subjects were reviewed by an experienced neuroradiologist, who scored the severity of white matter hyperintensities based on Scheltens scale. ${ }^{17}$ Briefly, the Scheltens scale is a semiquantitative measure of white matter lesion severity. In this study, we modified the Scheltens scale to 3 categories: 0 , if the subject had no white matter lesions; 1 , mild disease, if the subject had less than or equal to 5 white matter lesions, each measuring less than or equal to $3 \mathrm{~mm}$; and 2 , moderate disease, if the subject had more than 5 white matter lesions or lesions measuring greater than $3 \mathrm{~mm}$.

Maps for FA and the 3 orthogonal tensor eigenvalues $\left(\lambda_{1}, \lambda_{2}, \lambda_{3}\right)$ were created from the raw data using the FMRIB software library (FSL version 3.3). This set of tools was used to perform eddy current correction, skull-stripping, and voxelwise diffusion tensor reconstruction. For further details regarding processing, please see http://www.fmrib.ox.ac.uk/fsl/.

\section{ROI Analysis}

ROI analysis was performed using MRIcro (http://www.cabiatl.com/ mricro/) in batches by a single operator (G.C.C.), who was blinded to apoE status and clinical information. ROIs were placed on the native FA maps in the anterior and posterior corpus callosum, the bilateral anterior and posterior cingulate white matter, and the bilateral parahippocampal white matter (Fig 1). The following landmarks were used for ROI placement. In the axial plane, the genu and splenium were used for anterior/posterior corpus callosum ROI placement, and the parahippocampal white matter ROIs were placed at the level of the midbrain. In the coronal plane, ROIs were placed in the anterior and posterior cingulate white matter, anterior and posterior to the level of the corticospinal tracts. Each ROI encompassed approximately 3-6 voxels. The ROI measurements were repeated after 3 months, yielding the following intraclass correlation coefficients: anterior corpus callosum 0.88 , posterior corpus callosum 0.81 , right parahippocampal white matter 0.94 , left parahippocampal white matter 0.95 , right anterior cingulate white matter 0.94 , left anterior cingulate white matter 0.92 , right posterior cingulate white matter 0.91 , left posterior cingulate white matter 0.92 . Since there were no significant differences between left and right measurements $(P>.05)$, the bilateral measurements were averaged in the analysis. The same ROIs were then applied to each eigenvalue map to examine differences in mean, axial, and radial diffusivity.

Ordinary least squares regressions, with age as a covariate, were used to assess for statistical differences in FA, MD, axial diffusivity, and radial diffusivity between the apoE $\varepsilon 2$ and apoE $\varepsilon 3$ groups. A $P$ value of less than 0.05 was considered statistically significant. Cohen $d$ effect sizes were also calculated using the following formula: $d=$ (mean 1 - mean2)/pooled standard deviation. 


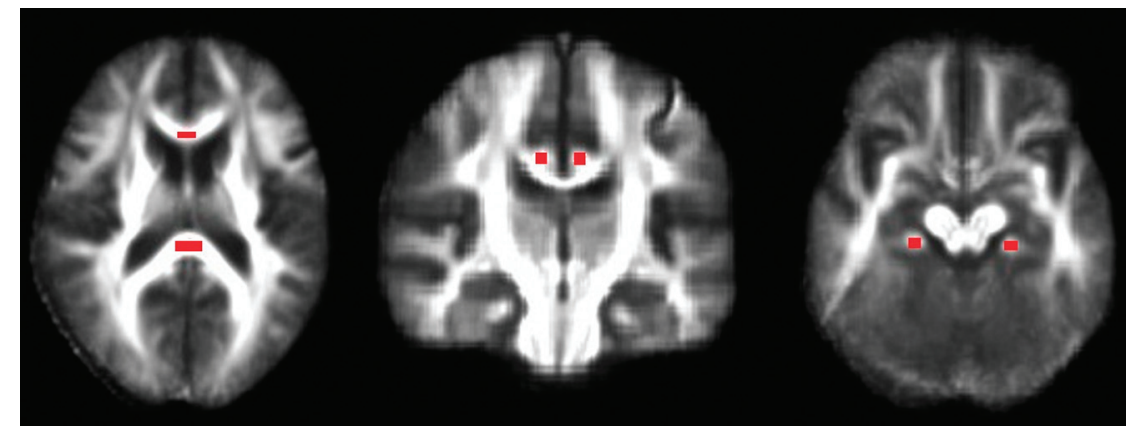

Fig 1. ROls (red) manually delineated on fractional anisotropy maps: anterior/posterior corpus callosum, anterior/posterior cingulate white matter, and the parahippocampal gyrus white matter.

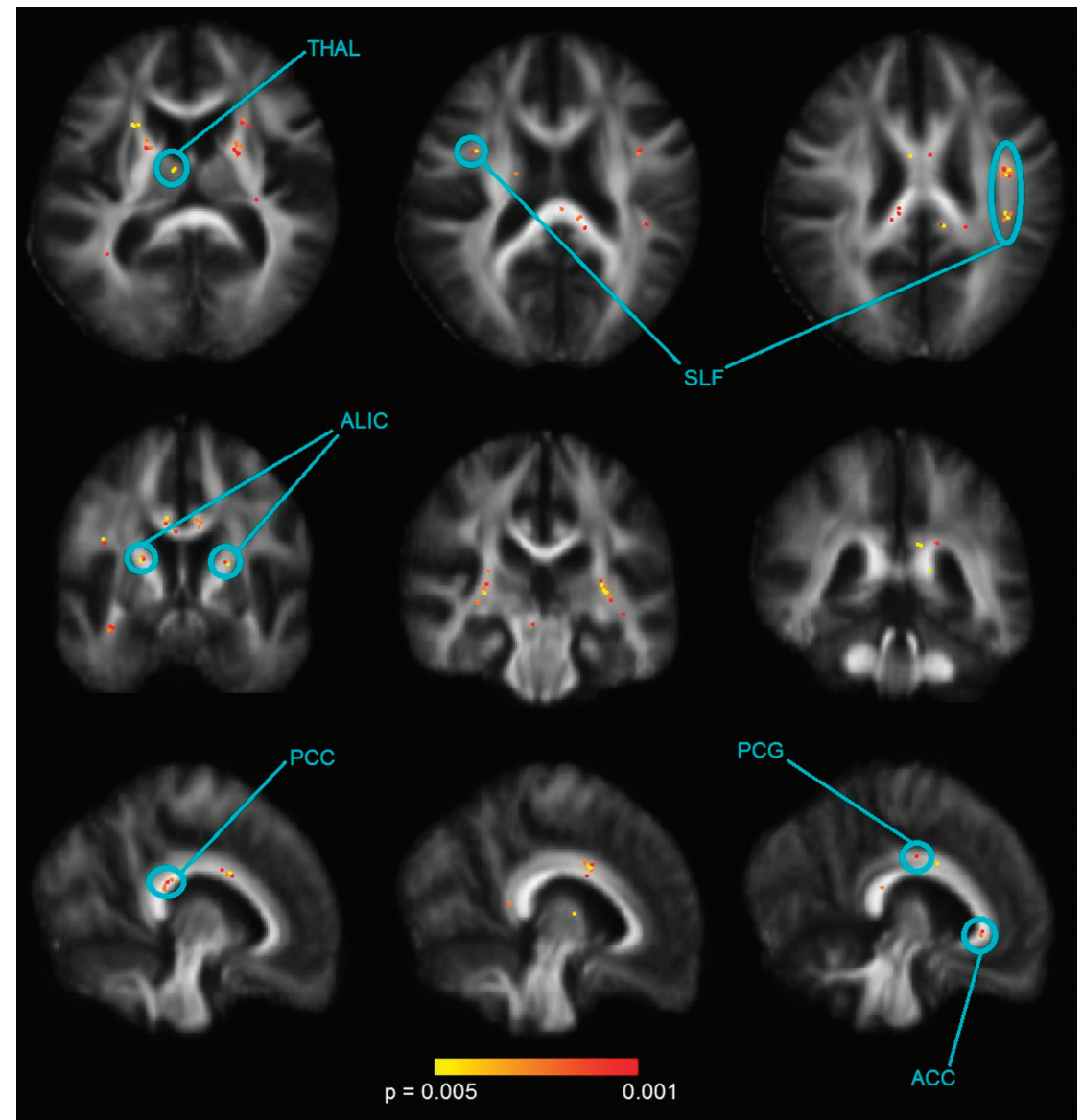

Fig 2. Tract-based spatial statistics demonstrates regions of higher FA in apoE $\varepsilon 2$ carriers relative to apoE $3 / 3$ controls $(P<.005$, uncorrected). THAL indicates thalamus; SLF $=$ superior Iongitudinal fasciculus; ALIC = anterior limb of the internal capsule; PCC = posterior corpus callosum; PCG $=$ posterior cingulate; $A C C=$ anterior corpus callosum

\section{Whole-Brain Analysis}

An operator-independent voxel-based approach, known as TBSS (version 1.0, http://www.fmrib.ox.ac.uk/fsl/tbss/), ${ }^{18}$ was used to interrogate the white matter between groups across the whole brain. Briefly, this approach first aligns each individual FA image to a common target FA image, using a 12-parameter affine transformation used in the FMRIB Linear Image Registration Tool. All the FA images are then affine-registered into standard $1 \times 1 \times 1 \mathrm{~mm}$ Montreal Neurologic Institute152 space. The registered FA images are subsequently merged into a single file to create a mean FA image for the group, which is then used to create a mean FA "skeleton." The purpose of this step is to define the white matter tracts that are common to all subjects in the analysis. The skeleton is thresholded at a partic- ular FA value, 0.3 in our study, to include the white matter tracts that are common to all subjects and most successfully aligned, as well as to exclude regions possibly containing gray matter, CSF, or misaligned voxels.

Each subject's aligned FA image is then projected onto the mean skeleton, and voxelwise statistics are performed between groups. For this exploratory analysis, we used a statistical significance threshold of $P<.005$, uncorrected for multiple comparisons.

\section{Results}

There were no significant differences in age, sex, MMSE, or white matter lesion load between the 2 groups (Table 1 ).

Consistent with our a priori hypothesis, the ROI approach 
demonstrated that apoE $\varepsilon 2$ carriers had higher FA in the posterior cingulate white matter relative to apoE $\varepsilon 3 / \varepsilon 3$ controls $(P=.01)$, yielding a Cohen $d$ effect size of 0.87 . apoE $\varepsilon 2$ carriers also demonstrated higher FA in the anterior corpus callosum $(P=.005)$, yielding a Cohen $d$ effect size of 0.95 . In contrast, there were no significant differences in FA in the posterior corpus callosum, anterior cingulate white matter, or parahippocampal white matter between apoE groups. In addition to higher FA, the apoE $\varepsilon 2$ carriers demonstrated lower radial diffusivity in the posterior cingulate white matter $(P=$ $.01)$ and anterior corpus callosum $(P=.03)$ ROIs relative to the apoE $\varepsilon 3 / \varepsilon 3$ group. No significant differences in mean or axial diffusivity were detected in the ROIs between apoE groups.

The whole-brain TBSS analysis detected regions of higher FA in the apoE $\varepsilon 2$ group compared with controls in the bilateral anterior thalamic radiations, corticopontine tracts, superior longitudinal fasciculus, and right thalamus, in addition to the cingulate white matter and corpus callosum $(P<$ .005) (Fig 2). ${ }^{19}$ Again, there were no regions in which the apoE $\varepsilon 3 / \varepsilon 3$ group demonstrated higher FA than the apoE $\varepsilon 2$ group.

\section{Discussion}

This study demonstrates that a single AD-protective apoE $\varepsilon 2$ allele is associated with regional differences in white matter microstructure in subjects who lack clinical evidence of cognitive impairment. The major findings were that 1) apoE $\varepsilon 2$ carriers had higher FA in the posterior cingulate white matter and corpus callosum, consistent with a different white matter fiber organization that probably reflects greater integrity; 2) apoE $\varepsilon 2$ carriers also demonstrated higher FA in association tracts, such as the superior longitudinal fasciculus and anterior limb of the internal capsule; and 3) the higher FA reflected lower radial diffusivity with no apparent differences in mean or axial diffusivity, which is believed to reflect stronger myelin integrity without gross tissue loss. Taken together, these findings suggest that the apoE $\varepsilon 2$ polymorphism is associated with more robust microstructural white matter integrity, which might serve as an index for greater resistance to AD pathology. Alternatively, due to the known protective effect of the apoE $\varepsilon 2$ polymorphism and the wide age range of participants, the more robust microstructural integrity could reflect decreased preclinical $\mathrm{AD}$ in the apoE $\varepsilon 2$ group relative to the apoE $\varepsilon 3 / \varepsilon 3$ carriers.

The finding of higher FA in the posterior cingulate white matter and corpus callosum of apoE $\varepsilon 2$ carriers, as evidenced by our ROI and whole-brain approaches, mirrors the pattern reported in prior literature comparing patients with $\mathrm{AD}$ and healthy individuals. ${ }^{7,8}$ The posterior cingulate is an integral component of the episodic memory network, receives major efferent tracts from the hippocampus, and is believed to function in memory retrieval. ${ }^{20,21}$ Furthermore, decreased FA in posterior cingulate white matter tracts and hypometabolism of the posterior cingulate cortex are early findings of $\mathrm{AD}$, which have also been seen in subjects with mild cognitive impairment and asymptomatic apoE $\varepsilon 4$ carriers with increased genetic risk for $\mathrm{AD} .^{7,8,22,23}$ Hence, it is not surprising that more robust posterior cingulate white matter, reflected by higher
$\mathrm{FA}$, may be associated with genetic resistance against $\mathrm{AD}$, as in our apoE $\varepsilon 2$ carrier group.

Alterations in callosal fibers have also been associated with $\mathrm{AD}$, which some studies have reported in both the anterior and posterior corpus callosum, ${ }^{11,24}$ whereas others only in the anterior $^{25}$ or posterior corpus callosum. ${ }^{7,26,27}$ Whether studies report adverse effects in the anterior or posterior corpus callosum could be technical in nature. For example, our ROI approach found higher FA in the anterior corpus callosum in apoE $\varepsilon 2$ carriers, whereas the TBSS approach found significant voxels in both the anterior and posterior corpus callosum. It is likely that differences at the voxel level, as measured using TBSS approach, are more sensitive than averaging FA values across an anatomically defined ROI approach, thereby diluting group differences. On the other hand, whole-brain template approaches, including TBSS, are potentially susceptible to image misregistrations, which may mimic FA differences. One study also proposed that lower FA in different areas of the corpus callosum could be explained by different mechanisms ${ }^{24}$; anterior callosal white matter changes could be secondary to retrogenesis, ${ }^{28}$ in which late-myelinating tracts are most susceptible to AD pathology early on, whereas posterior callosal changes could be secondary to wallerian degeneration related to neuronal loss. ${ }^{29}$ Similarly, asymptomatic apoE $\varepsilon 4$ carriers have exhibited lower FA in the anterior corpus callosum, possibly secondary to accelerated age-related loss, ${ }^{30}$ and in the posterior corpus callosum, possibly related to $\mathrm{AD}$ pathology in the temporoparietal cortex. ${ }^{14}$ Either protective effects of apoE $\varepsilon 2$ or decreased AD-related changes in the temporal and parietal lobes may explain the higher callosal FA in our study.

The exploratory whole-brain approach uncovered additional regions in which apoE $\varepsilon 2$ carriers demonstrated higher FA, all of which involved association fiber tracts - namely, the superior longitudinal fasciculus bilaterally, right thalamus, and the anterior limb of the internal capsule. The superior longitudinal fasciculus includes 3 bundles of long association fiber tracts, which connect the parietal lobes to the prefrontal cortex, ${ }^{31}$ and has been reported to be involved in $\mathrm{AD}{ }^{25,27,32}$ The thalamus has also been reported to be involved early in $\mathrm{AD}^{32,33}$ and serves as the gateway for connections between the cortex and brain stem. Finally, we found significant voxels in the corticothalamic fibers passing through the anterior limb of the internal capsule, which connect the anterior, medial dorsal, and lateral dorsal thalamic nuclei to the cingulate gyrus, limbic cortex, and prefrontal association cortex. ${ }^{34}$ Involvement of these fibers has been reported in prior studies comparing patients with $\mathrm{AD}$ and controls. ${ }^{25,32}$ Evidence for voxelwise differences in these areas among apoE $\varepsilon 2$ carriers is compatible with the concept of apoE $\varepsilon 2$ being resistant to $\mathrm{AD}$.

The finding that apoE $\varepsilon 2$ carriers have higher FA in certain white matter tracts on the basis of lower radial diffusivity, without concomitant changes in axial diffusivity, suggests more robust myelination as a potential mechanism. Indeed, animal studies of experimentally induced myelin loss, ${ }^{5,6,35}$ and a human study following late demyelination after surgical corpus callosotomy, ${ }^{36}$ have demonstrated decreases in FA with increased radial, and no change in axial, diffusivity. This pattern of decreased FA and increased radial diffusivity has also been reported among nondemented women carrying the 
apoE $\varepsilon 4$ risk allele and a family history of $\mathrm{AD}^{37}$ and has been associated with higher cerebrospinal total tau, ${ }^{38}$ which suggests that myelin disruption may represent an early pathogenic process of AD. ${ }^{39}$ Postmortem neuropathologic studies have found white matter changes in approximately $60 \%$ of patients with $\mathrm{AD},{ }^{29}$ with several studies reporting demyelination or myelin damage from beta-amyloid in these patients. ${ }^{30,40-42}$ Taken together, one hypothesis for the higher FA and lower radial diffusivity in our apoE $\varepsilon 2$ carriers is that the more robust myelination reflects less toxic demyelination from underlying Alzheimer pathology. Indeed, several studies have reported decreased amyloid among apoE $\varepsilon 2$ carriers. ${ }^{43,44}$ An alternative explanation is that apoE $\varepsilon 2$ carriers are less susceptible to $\mathrm{AD}$ due to intrinsically more robust myelination. One prior study has reported higher synaptophysin among apoE $\varepsilon 2$ carriers, which is a marker of synaptic integrity. ${ }^{45}$ Furthermore, a study in apoE $\varepsilon 2$ targeted replacement mice demonstrated longer dendritic spines and increased arborization. $^{46}$

This study has several limitations. First, this is a case control study in which major confounders that are often reported in $\mathrm{AD}$ literature-such as age, sex, cognitive function, and concomitant vascular disease reflected by white matter lesions-were controlled; however, unforeseenable confounders are possible. For example, family history of $\mathrm{AD}$ would be another possible confounder and was not elicited in this study. There is evidence in the literature to suggest that apoE $\varepsilon 2$ carriers have slower rates of hippocampal atrophy ${ }^{44}$ and slower rates of cognitive decline, ${ }^{47}$ though longitudinal monitoring of our particular cohort would be necessary to validate our findings as a true mechanism of AD protection. Furthermore, validation of DTI as a robust biologic parameter has yet to be performed. In particular, white matter lesion load may interfere with DTI metrics, though there was no significant difference in lesion load at the group level. Because of small sample size, our TBSS results were uncorrected for multiple comparisons and remain exploratory. Nevertheless, this study indicates that the apoE $\varepsilon 2$ polymorphism has a biologic underpinning in microstructural white matter integrity and lends further support to DTI metrics serving as promising biomarkers of future AD risk.

This work was funded by the National Institutes of Health, National Institute of Biomedical Imaging and Bioengineering (T32 EB001631-05), and NIH grant R01 AG010897. Drs. Chiang and Zhan report no disclosures. Dr. Schuff received honorarium from the Michael J. Fox Foundation, the British Research Council, and the Elsevier Publishing Company; he also receives research support from the Michael J. Fox Foundation, Department of Defense (WX), P41 RR023953 (coinvestigator); P50AG23501 (coinvestigator). Dr. Weiner serves on scientific advisory boards for Bayer Schering Pharma, Eli Lilly, Nestle, CoMentis, Neurochem, Eisai, Avid, Aegis, Genentech, Allergan, Lippincott, Bristol Meyers Squibb, Forest, Pfizer, McKinsey, Mitsubishi, and Novartis. He has received non-industry-supported funding for travel; serves on the editorial board of Alzheimer's \& Dementia; received honoraria from the Rotman Research Institute and BOLT International; receives research support from Merck \& Co, Avid, NIH [U01AG024904 (PI), P41 RR023953 (PI), R01 AG10897 (PI), P01AG19724 (Coinvestigator), P50AG23501(Coinvestigator), R24 RR021992 (Coinvestigator), R01 NS031966 (Coinvestigator), and P01AG012435 (Coinvestigator)], the Department of Defense [DAMD17-01-01-0764 (PI)], and the Veterans Administration [MIRECC VISN 21 (Core PI)]; and holds stock in Synarc and Elan Pharmaceuticals.

Additional Disclosures: Norbert Schuff-Consultancy: NIH, Irish Research Council, Comments: Grant reviews; Expert Testimony: Kohn Kohn \& Colapinto, LLP, Comments: Expert witness on MRl; Grants/Grants Pending: $\mathrm{NIH}^{*}$ Department of Defense, ${ }^{*}$ Michael J. Fox Foundation for Parkinson's Research;* Payment for Development of Educational Presentations: Parkinson's Institute Sunnyvale, Comments: Lectures MRI in Parkinsons disease.
Michael Weiner-UNRELATED: Consultancy: Elan/Wyeth, Novartis, Forest, Ipsen, Daiichi Sankyo, Astra Zeneca, Araclon, Medivation/Pfizer, TauRx Therapeutics, LTD, Bayer Healthcare, Biogen Idec, Exonhit Therapeutics, SA, Servier, Synarc; Payment for Lectures (including Service on Speakers Bureaus): Various speaking engagements at universities and society meetings. (*Money paid to institution.)

\section{References}

1. Corder EH, Saunders AM, Risch NJ, et al. Protective effect of apolipoprotein E type 2 allele for late onset Alzheimer disease. Nat Genet 1994;7:180-84

2. Seshadri S, Drachman DA, Lippa CF. Apolipoprotein E epsilon 4 allele and the lifetime risk of Alzheimer's disease: what physicians know, and what they should know. Arch Neurol 1995;52:1074-79

3. Thakkinstian A, Bowe S, McEvoy M, et al. Association between apolipoprotein E polymorphisms and age-related macular degeneration: a HuGE review and meta-analysis. Am J Epidemiol 2006;164:813-22

4. Le Bihan D, Mangin J, Poupon C, et al. Diffusion tensor imaging: concepts and applications. J Magn Reson Imaging 2001;13:534-46

5. Song SK, Sun SW, Ju WK, et al. Diffusion tensor imaging detects and differentiates axon and myelin degeneration in mouse optic nerve after retinal ischemia. Neuroimage 2003;20:1714-22

6. Song SK, Sun SW, Ramsbottom MJ, et al. Dysmyelination revealed through MRI as increased radial (but unchanged axial) diffusion of water. Neuroimage 2002;17:1429-36

7. Zhang Y, Schuff N, Jahng GH, et al. Diffusion tensor imaging of cingulum fibers in mild cognitive impairment and Alzheimer disease. Neurology 2007;68:13-19

8. Zhou Y, Dougherty JH, Hubner KF, et al. Abnormal connectivity in the posterior cingulate and hippocampus in early Alzheimer's disease and mild cognitive impairment. Alzheimers Dement 2008;4:265-70

9. Fellgiebel A, Wille P, Muller MJ, et al. Ultrastructural hippocampal and white matter alterations in mild cognitive impairment: a diffusion tensor imaging study. Dement Geriatr Cogn Disord 2004;18:101-08

10. Salat DH, Tuch DS, van der Kowe AJW, et al. White matter pathology isolates the hippocampal formation in Alzheimer's disease. Neurobiol Aging 2010;31: 244-56

11. Bozzali M, Falini A, Franceschi M, et al. White matter damage in Alzheimer's disease assessed in vivo using diffusion tensor magnetic resonance imaging. J Neurol Neurosurg Psychiatry 2002;72:742-46

12. Huang J, Friedland RP, Auchus AP. Diffusion tensor imaging of normalappearing white matter in mild cognitive impairment and early Alzheimer's disease: preliminary evidence of axonal degeneration in the temporal lobe. AJNR Am J Neuroradiol 2007;28:1943-48

13. Nierenberg J, Pomara N, Hoptman MJ, et al. Abnormal white matter integrity in healthy apolipoprotein E epsilon4 carriers. NeuroReport 2005;16:1369-72

14. Persson J, Lind J, Larsson A, et al. Altered brain white matter integrity in healthy carriers of the apoE epsilon4 allele: a risk for AD? Neurology 2006;66:1029-33

15. Smith $\mathrm{CD}$, Chebrolu $\mathrm{H}$, Andersen $\mathrm{AH}$, et al. White matter diffusion alterations in normal women at risk of Alzheimer's disease. Neurobiol Aging 2010;31: 1122-31

16. Folstein MF, Folstein SE, McHugh PR. "Mini-mental state." A practical method for grading the cognitive state of patients for the clinician. JPsychiatr Res 1975;12:189-98

17. Scheltens $\mathrm{P}$, Barkhof F, Leys D, et al. A semiquantative rating scale for the assessment of signal hyperintensities on magnetic resonance imaging. $\mathrm{J} \mathrm{Neu}$ rol Sci 1993;114:7-12

18. Smith SM, Jenkinson M, Johansen-Berg H, et al. Tract-based spatial statistics: Voxelwise analysis of multi-subject diffusion data. NeuroImage 2006;31:1487505

19. Mori S, Wakana S, Nagae-Poetscher LM, et al. MRI Atlas of Human White Matter. Amsterdam: Elsevier; 2005

20. Duvernoy H. The Human Hippocampus. 2nd ed. Berlin: Springer-Verlag; 1998:34

21. Valenstein E, Bowers D, Verfaellie M, et al. Retrosplenial amnesia. Brain 1987;110:1631-46

22. Minoshima S, Foster NL, Kuhl DE. Posterior cingulate cortex in Alzheimer's disease. Lancet 1994;344:895

23. Reiman EM, Caselli RJ, Yun LS, et al. Preclinical evidence of Alzheimer's disease in persons homozygous for the epsilon 4 allele for apolipoprotein $\mathrm{E}$. N Engl J Med 1996;334:752-58

24. Di Paola M, Di Iulio F, Cherubini A, et al. When, where, and how the corpus callosum changes in MCI and AD. Neurology 2010;74:1136-42

25. Xie S, Xiao JX, Gong GL, et al. Voxel-based detection of white matter abnormalities in mild Alzheimer disease. Neurology 2006;66:1845-49

26. Takahashi S, Yonezawa $\mathrm{H}$, Takahashi J, et al. Selective reduction of diffusion anisotropy in white matter of Alzheimer disease brains measured by 3.0 Tesla magnetic resonance imaging. Neurosci Lett 2002;332:45-48

27. Rose SE, Chen F, Chalk JB, et al. Loss of connectivity in Alzheimer's disease: an 
evaluation of white matter tract integrity with colour coded MR diffusion tensor imaging. J Neurol Neurosurg Psychiatry 2000;69:528-30

28. Reisberg B, Franssen EH, Souren LE, et al. Evidence and mechanisms of retrogenesis in Alzheimer's and other dementias; management and treatment import. Am J Alzheimers Dis Other Demen 2002;17:202-12

29. Brun A, Englund E. A white matter disorder in dementia of the Alzheimer type: a pathoanatomical study. Ann Neurol 1986;19:253-62

30. Filippini N, Zarei M, Beckmann CF, et al. Regional atrophy of transcallosal prefrontal connections in cognitively normal apoE epsilon4 carriers. J Magn Reson Imaging 2009;29:1021-26

31. Schmahmann JD, Pandya DN. Fiber Pathways of the Brain. Oxford: Oxford University Press; 2006:133-35

32. Medina D, DeToledo-Morell L, Urresta F, et al. White matter changes in mild cognitive impairment and AD: a diffusion tensor imaging study. Neurobiol Aging 2006;27:663-72

33. Rose SE, McMahon KL, Janke AL, et al. Diffusion indices on magnetic resonance imaging and neuropsychological performance in amnestic mild cognitive impairment. J Neurol Neurosurg Psychiatry 2006;77:1122-28

34. Wiegell MR, Tuch DS, Larsson HBW, et al. Automatic segmentation of thalamic nuclei from diffusion tensor magnetic resonance imaging. Neuroimage 2003;19:391-401

35. Sun SW, Liang HF, Trinkaus K, et al. Noninvasive detection of cuprizone induced axonal damage and demyelination in the mouse corpus callosum. Magn Reson Med 2006;55:302-08

36. Concha L, Gross DW, Wheatley BM, et al. Diffusion tensor imaging of timedependent axonal and myelin degradation after corpus callosotomy in epilepsy patients. Neuroimage 2006;32:1090-99

37. Gold BT, Powell DK, Andersen AH, et al. Alterations in multiple measures of white matter integrity in normal women at high risk for Alzheimer's disease. Neuroimage 2010;52:1487-94

38. Stenset V, Bjornerud A, Fjell AM, et al. Cingulum fiber diffusivity and CSF T-tau in patients with subjective and mild cognitive impairment. Neurobio Aging 2011;32:581-89.

39. Bartzokis G. Alzheimer's disease as homeostatic responses to age-related myelin breakdown. Neurobiol Aging 2011;32:1341-71.

40. Sjobeck M, Haglund M, Englund E. Decreasing myelin density reflected increasing white matter pathology in Alzheimer's disease-a neuropathological study. Int J Geriatr Psychiatry 2005;20:919-26

41. Wang DS, Bennett DA, Mufson EJ, et al. Contribution of changes in ubiquitin and myelin basic protein to age-related cognitive decline. Neurosci Res 2004 48:93-100

42. Roher AE, Weiss $\mathrm{N}$, Kokjohn $\mathrm{TA}$, et al. Increased A beta peptides and reduced cholesterol and myelin proteins characterize white matter degeneration in Alzheimer's disease. Biochemistry 2002;41:11080-90

43. Lippa CF, Smith TW, Saunders AM, et al. Apolipoprotein E-epsilon 2 and Alzheimer's disease: genotype influences pathologic phenotype. Neurology 1997;48:515-19

44. Chiang GC, Insel PS, Tosun D, et al. Hippocampal atrophy rates and CSF biomarkers in elderly apoE2 normal subjects. Neurology 2010;75:1976-81

45. Berlau DJ, Corrada MM, Head E, et al. apoE epsilon2 is associated with intact cognition but increased Alzheimer pathology in the oldest old. Neurology 2009;72:829-34

46. Dumanis SB, Tesoriero JA, Babus LW, et al. ApoE 4 decreases spine density and dendritic complexity in cortical neurons in vivo. J Neurosci 2009;29:15317-22

47. Wilson RS, Bienias JL, Berry-Kravis E, et al. The apolipoprotein E epsilon 2 allele and decline in episodic memory. J Neurol Neurosurg Psychiatry 2002;73: $672-77$ 